\title{
Anatomical sites (Takasaki's segmentation) predicts the recurrence-free survival of hepatocellular carcinoma
}

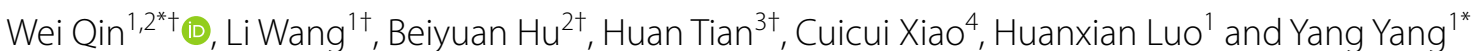

\begin{abstract}
Background: Until now, several classification staging system and treatment algorithm for hepatocelluar carcinoma (HCC) has been presented. However, anatomical location is not taken into account in these staging systems. The aim of this study is to investigate whether anatomical sites could predict the postoperative recurrence of HCC patients.

Methods: 294 HCC patients were enrolled in this retrospective study. A novel score classification based on anatomical sites was established by a Cox regression model and validated in the internal validation cohort.

Results: HCC patients were stratified according to the novel score classification into three groups (score 0 , score 1-3 and score 4-6). The predictive accuracy of the novel recurrence score for HCC patients as determined by the area under the receiver operating characteristic curves (AUCs) at 1, 3, and 5 years (AUCs 0.703, 0.706, and 0.605) was greater than that of the other representative classification systems. These findings were supported by the internal validation cohort. For patients with Barcelona Clinic Liver Cancer (BCLC) 0 and A stage, our data demonstrated that there was no significant difference in recurrence-free survival (RFS) between patients with score 0 and liver transplantation recipients. Additionally, we introduced this novel classification system to guide anatomical liver resection for centrally located liver tumors.
\end{abstract}

Conclusion: The novel score classification may provide a reliable and objective model to predict the RFS of HCC after hepatic resection.

Keywords: Hepatocelluar carcinoma, Recurrence-free survival, Takasaki's segmentation

\section{Background}

Hepatocellular carcinoma (HCC) is the fifth most prevalent malignancy worldwide, with approximately 750,000 new cases diagnosed annually [1]. In the worldwide, about $78 \%$ of $\mathrm{HCC}$ patients are correlated with hepatitis $B$ virus (HBV) and hepatitis $C$ virus (HCV) infection [2]. Due to heterogeneity of the patient population and low

\footnotetext{
*Correspondence: qw9911022@hotmail.com; yysysu@163.com

†Wei Qin, Li Wang, Beiyuan Hu and Huan Tian contributed equally to this study

${ }^{1}$ Department of Hepatic Surgery, The Third Affiliated Hospital of Sun YatSen University, 600 Tianhe Road, Guangzhou 510630, China

Full list of author information is available at the end of the article
}

utilization of HCC screening, only $10-37 \%$ of patients are candidates for surgical resection at initial HCC diagnosis [3-5]. Approximately $70 \%$ of patients with $\mathrm{HCC}$ develop recurrence within 5 years after curative resection [6]. Although many previous studies have reported that the recurrence is associated with tumor biological characteristics, such as large tumor size, multiple tumors, poor differentiation, macro- and microvascular invasion, satellite lesions, liver conditions and sex difference [6-8], the impact of HCC tumor location on recurrence after hepatic resection (HR) is still poorly understood. Until now, only one retrospectively study has been indicated that in HCC patients with multifocal tumors meeting 
the Milan criteria, tumors located in the same hepatic section (Couinaud's segmentation) may lead to better long-term survival and lower HCC recurrence rates than tumors in different sections after HR [9].

According to the Glissonean pedicle classification as described by Takasaki, the hepatoduodenal ligament forms the main trunk of the tree of the Glissonean pedicle, which expands into two branches (the right and left primary branches) at the hepatic hilum. The right branch is subdivided into two secondary branches, whereas the left branch continues as a transverse portion with a secondary branch. Consequently, the liver can be separated into three segments (a left, a middle and a right), which is supplied directly from the primary branch, and the caudate [10]. This Glissonean pedicle approach has made different types of hepatectomy possible including not only hemihepatectomy but also small anatomical hepatectomies, such as sectionectomy and Couinaud's segmentectomy in a cirrhotic liver [11].

Here, we further investigated the impact of tumor location (Takasaki's classification) and exclusively established a novel classification system to predict recurrence-free survival (RFS) of HCC patients.

\section{Methods}

\section{Staging systems}

Several systems have been proposed for staging HCC, including Barcelona Clinic Liver Cancer (BCLC), HongKong Liver Cancer (HKLC), American Joint Committee on Cancer (AJCC, TNM $8^{\text {th }}$ ) and HKLC staging systems [12-14]. According to BCLC staging system, BCLC Stage 0 , single nodular $<2 \mathrm{~cm}, \mathrm{BCLC}$ Stage $\mathrm{A}$, single nodular or 2-3 tumors with a maximum diameter $<3 \mathrm{~cm}$, BCLC Stage B, multinodular, BCLC Stage C, any tumor with radiologically evident and/or histologically proven portal invasion [15].

\section{Patients and study design}

In our study, we retrospectively analyzed 241 patients without any preoperative treatment who underwent resection of HCC with curative intent from the Third Affiliated Hospital of Sun Yat-sen University between January 2007 and January 2017. 53 HCC patients with BCLC 0 and A stage were received liver transplantation (LT) at the Third Affiliated Hospital of Sun Yat-sen University during a period from May 2012 to August 2016. The diagnosis of HCC was confirmed by pathological examination in all cases.

\section{Patient selection and operative indications}

The choice of surgical treatment was dependent on comprehensive assessment of preoperative imaging studies, intraoperative ultrasonography, tumor characteristics, remnant liver volume and underlying liver condition. To determine the size, nodule number, location of tumor, and its relationship with adjacent vital liver vasculature, all patients were examined by routine preoperative assessment, including abdominal ultrasonography, highresolution, contrast-enhanced computed tomography (CT) and/or magnetic resonance imaging (MRI). Only patients with BCLC 0-C stage were included. The patients with or without cirrhosis whose the remnant liver volume evaluated by CT or MRI $>50 \%$ or $>30 \%$ were considered for liver resection $[16,17]$. Liver functional reserve was assessed by the Child-Pugh classification and liver function tests. Live resection was indicated only for patients with compensated liver function (Child-Pugh grade A or B). Routine preoperative assessment also included chest radiograph, electrocardiogram, renal function tests, whole blood count, and coagulation profile.

HCC patients who underwent LT were also included in this study. Patients were received preoperative imaging examination, liver function test, and routine preoperative assessment before LT.

\section{Surgical procedure for hepatectomy}

Hepatectomy was carried out via a bilateral subcostal incision with a midline extension or a J-shaped incision in the right upper abdomen. Intraoperative ultrasonography was performed routinely to locate tumors, assess resectability of the tumors, and detect lesions not apparent on preoperative radiology. To reduce ischemia-reperfusion injury to the remnant liver, selective hepatic inflow occlusion was performed intermittently [18]. Once it was difficult to isolate the right/left portal pedicle en bloc, Pringle's maneuver was recommended. Finally, the dissection surface was scrutinized for bleeding or bile leakage before closing the abdominal wall.

\section{Surgical procedure for LT and immunosuppressants}

All patients were received piggyback LT. The immunosuppression regimen was consisted of anti-interleukin-2 (basiliximab) induction therapy and tacrolimus/ sirolimus-based therapy in combination with mycophenolate mofetil.

\section{Data collection}

Clinicopathologic variables including sex, age at resection, Child-Pugh grading and preoperative $\alpha$-fetoprotein (AFP) level were collected. Liver cirrhosis was confirmed by histopathologic examination. Tumor pathologic, surgical and perioperative data including tumor size, tumor nodule number, presence of microscopic vascular invasion, tumor differentiation, duration of surgery, intraoperative blood loss, presence of intraoperative blood transfusion and operative complications were also 
collected. HCC located in multiple segments (MultipleHCC) was defined as tumor located in two or more Takasaki's segments, whatever the tumor nodule number was. The multiple-HCC tumor size was calculated as the maximum size of each individual tumor.

\section{Follow-up studies}

RFS was defined as the time from the day of operation to the date when recurrence was first diagnosed or last follow-up. Physical examination, liver function tests, serum AFP, ultrasonography, chest X-ray, and CT and/or MRI were performed once every 3 months for the first 2 years and then twice a year thereafter. The treatment of choice for HCC recurrence was dependent on the number and location of the recurrent tumors, and the liver condition including repeat hepatectomy, LT, radiofrequency ablation (RFA) and transcatheter arterial chemoembolization (TACE).

\section{Statistical analysis}

All statistical analyses were performed with the IBM SPSS 19.0 statistical software (SPSS, Armonk, USA). Continuous variables were presented as mean \pm standard deviation (SD). Categorical variables were compared using the $\chi^{2}$ test. RFS was estimated by Kaplan-Meier analysis. To establish the novel score, the regression coefficients (B-values) of the Cox regression model were multiplied by 2 and rounded to the nearest unit to obtain simple point numbers in this study. To further evaluate the discriminative ability of this novel score in predicting RFS of HCC patients, the area under the receiver operating characteristic curves (AUCs) of the novel score was compared with that of the other representative classification systems. $P<0.05$ was considered statistically significant.

\section{Results}

\section{Clinicopathologic characteristics and perioperative data}

Clinicopathologic baseline data of 241 patients underwent liver resection were illustrated in Table 1 . According to Takasaki's segmentation, there were 64 patients with HCC located in the left segment (L-HCC), 28 patients with HCC located in the middle segment (M-HCC), 58 patients with HCC located in the right segment (R-HCC), 1 patient with HCC located in caudate area and 60 multiple-HCC patients in the training cohort Table 2. In the internal validation cohort, there were $4 \mathrm{~L}-\mathrm{HCC}$, $12 \mathrm{M}-\mathrm{HCC}, 10 \mathrm{R}-\mathrm{HCC}, 1$ patient with HCC located in caudate area and 3 multiple-HCC patients.

Perioperative outcomes of HCC patients in the training cohort were shown in Table 3. Our data showed that the multiple segments-HCC group had a larger tumor size than the simple segments-HCC group $(P=0.002)$.
Table 1 Clinical characteristics of 241 HCC patients underwent hepatectomy

\begin{tabular}{lll}
\hline Variables & $\begin{array}{l}\text { Training cohort } \\
(\mathbf{n}=\mathbf{2 1 1})\end{array}$ & $\begin{array}{l}\text { Internal } \\
\text { validation } \\
\text { cohort }(\mathbf{n}=\mathbf{3 0})\end{array}$ \\
\hline $\begin{array}{ll}\text { Age (years) } \\
\text { Gender, M: F }\end{array}$ & $50.0 \pm 11.4$ & $50.0 \pm 13.1$ \\
HBsAg & $200: 11$ & $26: 4$ \\
Positive & & \\
Negative & $203(96.2 \%)$ & $28(93.3 \%)$ \\
AFP (ng/ml) & $8(3.8 \%)$ & $2(6.7 \%)$ \\
Platelet count $\left(10^{9} / \mathrm{L}\right)$ & $380.4 \pm 519.4$ & $326.3 \pm 455.2$ \\
Prothrombin time $(\mathrm{s})$ & $175.3 \pm 78.8$ & $202.3 \pm 86.6$ \\
Total bilirubin $(\mu \mathrm{mol} / \mathrm{L})$ & $13.6 \pm 1.3$ & $13.5 \pm 1.3$ \\
Albumin $(\mathrm{g} / \mathrm{L})$ & $17.6 \pm 13.2$ & $14.7 \pm 9.4$ \\
Alanine aminotransferase $(\mathrm{U} / \mathrm{L})$ & $48.6 \pm 42.7$ & $39.6 \pm 4.8$ \\
Child-Pugh score & & $48.9 \pm 28.7$ \\
A & $210(99.5 \%)$ & $28(93.3 \%)$ \\
B & $1(0.5 \%)$ & $2(6.7 \%)$ \\
\hline
\end{tabular}

AFP alpha-fetoprotein, $\mathrm{HCC}$ hepatocellular carcinoma

Compared with the single segment-HCC group, the multiple segments-HCC group exhibited significantly more tumor nodules $(P<0.001)$. Furthermore, the multiple segments-HCC group had a longer operation time compared with the simple segments-HCC group $(P=0.017)$. Perioperative morbidity was categorized according to ClavienDindo classification. Three patients died in the hospital because of postoperative acute hepatic failure and peptic ulcer bleeding, resulting in a perioperative mortality rate of $1.4 \%$. Otherwise, the other parameters including capsulation formation, differentiation grade, microvascular invasion, liver cirrhosis, BCLC stage, blood loss, blood transfusion, surgical margin, overall complications and in-hospital mortality were comparable between the two groups.

\section{A novel score model based on tumor location}

At the time of censor of this study, there were 150 (150/211, 71.1\%) patients with recurrence of HCC in the training cohort. With regards to the site of recurrence, intrahepatic recurrence was the most common site and occurred in 136 patients (136/150, 90.7\%). Extrahepatic recurrence was diagnosed in 14 patients (14/150, 9.3\%). The 1-, 3-, and 5-years overall recurrence rates were $38.9 \%, 63.0 \%$ and $83.4 \%$, respectively. Our data showed a significant decrease in RFS rate for patients with M-HCC, especially 2 years after operative intervention. KaplanMeier estimates of the 1-, 3-, and 5-years RFS rates for M-HCC group were $80.9 \%, 66.8 \%$ and $53.2 \%$, respectively. However, the recurrence rates of patients with L-HCC, 
Table 2 Classification of the 211 HCC patients who underwent hepatectomy in the training cohort

\begin{tabular}{|c|c|c|c|c|c|c|}
\hline \multirow[t]{2}{*}{ Variables } & \multicolumn{4}{|c|}{ Single segment-HCC $(n=151)$} & \multirow{2}{*}{$\begin{array}{l}\text { Multiple } \\
\text { segments-HCC } \\
(n=60)\end{array}$} & \multirow{2}{*}{$\begin{array}{l}\text { Single segment vs } \\
\text { Multiple segment } \\
\text { (P-value) }\end{array}$} \\
\hline & $\begin{array}{l}\text { Left segment } \\
(n=64)\end{array}$ & $\begin{array}{l}\text { Middle } \\
\text { segment } \\
(n=28)\end{array}$ & $\begin{array}{l}\text { Right } \\
\text { segment } \\
(n=58)\end{array}$ & Caudate area $(n=1)$ & & \\
\hline Tumor size $(\mathrm{cm})$ & $6.5 \pm 4.8$ & $4.0 \pm 2.8$ & $4.8 \pm 2.5$ & 8.5 & $7.1 \pm 3.3$ & 0.002 \\
\hline Tumor nodule number & & & & & & $<0.001$ \\
\hline Single & $58(90.6 \%)$ & $24(85.7 \%)$ & $51(87.9 \%)$ & $1(100 \%)$ & $37(61.7 \%)$ & \\
\hline Multiple ( $\geq 2$ ) & $6(9.4 \%)$ & $4(14.3 \%)$ & $7(12.1 \%)$ & 0 & $23(38.3 \%)$ & \\
\hline Capsulation formation & & & & & & 0.540 \\
\hline Present & $39(60.9 \%)$ & $15(53.6 \%)$ & $34(58.6 \%)$ & 0 & $32(53.3 \%)$ & \\
\hline Absent & $25(39.1 \%)$ & $13(46.4 \%)$ & $24(41.4 \%)$ & $1(100 \%)$ & $28(46.7 \%)$ & \\
\hline Differentiation grade ${ }^{a}$ & & & & & & 0.801 \\
\hline$|-| \mid$ & $61(95.3 \%)$ & $25(89.3 \%)$ & $49(84.5 \%)$ & $1(100 \%)$ & $55(91.7 \%)$ & \\
\hline III-IV & $3(4.7 \%)$ & $3(10.7 \%)$ & $9(15.5 \%)$ & 0 & $5(8.3 \%)$ & \\
\hline $\mathrm{MVI}$ & & & & & & 1.000 \\
\hline Present & $18(28.1 \%)$ & $5(17.9 \%)$ & $16(27.6 \%)$ & 0 & $16(26.7 \%)$ & \\
\hline Absent & $46(71.9 \%)$ & $23(82.1 \%)$ & $42(72.4 \%)$ & $1(100 \%)$ & $44(73.3 \%)$ & \\
\hline Liver cirrhosis & & & & & & 0.715 \\
\hline Present & $53(82.8 \%)$ & $18(64.3 \%)$ & 44 (73.3\%) & 0 & $50(83.3 \%)$ & \\
\hline Absent & 11 (17.2\%) & $10(35.7 \%)$ & $14(26.7 \%)$ & $1(100 \%)$ & 10 (16.7\%) & \\
\hline BCLC stage & & & & & & 0.161 \\
\hline $0 \& A$ & 34 (53.1\%) & $20(71.4 \%)$ & 44 (73.3\%) & 1 (100\%) & 27 (45.0\%) & \\
\hline B & $3(4.7 \%)$ & 4 (14.3\%) & 0 & 0 & 16 (26.7\%) & \\
\hline$C$ & $27(42.2 \%)$ & $4(14.3 \%)$ & $14(26.7 \%)$ & 0 & $17(28.3 \%)$ & \\
\hline
\end{tabular}

$B C L C$ Barcelona Clinic Liver Cancer, MVI microvascular invasion, $H C C$ hepatocellular carcinoma

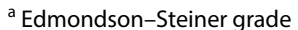

R-HCC and Multiple-HCC exhibited no significant difference (Additional file 1: Figure S1 and Additional file 3: Table S1).

Next, we performed univariate and multivariate analyses to evaluate the relationship between prognostic factors with RFS. Our data indicated that tumor location was a significant predictor of RFS (Additional file 4: Table S2). We built the risk score based on the regression coefficients weighted by the Cox model. The risk score was calculated as follows: score $=$ Tumor size $(<5 \mathrm{~cm}=0 ; \geq 5 \mathrm{~cm}=2)+$ Differentiation grade $(\mathrm{I}-\mathrm{II}=0$; III-IV $=2)+$ MVI $(-=0 ;+=1)+$ Tumor location (single segment $=0$; multiple segments $=1$ ).

\section{The novel score predicts RFS of HCC patients}

As was shown in Fig. 1A, there was no significant difference among score 1 , score 2 and score 3 in RFS. Furthermore, the median RFS of score 4, score 5 and score 6 was 17.5 months, 10.0 and 3.4 months, respectively. Accordingly, $211 \mathrm{HCC}$ patients were classified into score 0 , score $1-3$, and score 4-6 groups. The median RFS of the HCC patients with score 0 , score $1-3$, and score $4-6$ was 102.2 months (95\% CI, 90.9-113.5 months), 60.1 months
(95\% CI, 49.7-70.5 months) and 14.5 months (95\% CI, 6.8-22.2 months), respectively (Fig. 1B). Consistently, the performance of this novel score in RFS prediction was verified in the internal validation cohort (Fig. 1C).

Subsequently, we compared the accuracy of this novel score with that of the current commonly used staging systems, such as BCLC, HKLC, and TNM staging systems. Our data indicated that the AUCs of the novel score at 1,3 , and 5 years were $0.703,0.706$, and 0.605 , respectively, and were greater than those of the other three staging systems for HCC (Fig. 2A-C). In the internal validation cohort, the AUCs of our novel score at 1 , 3 , and 5 years were $0.715,0.748$, and 0.801 , respectively (Fig. 2D-F). Collectively, compared with the other three staging systems, the novel score had a better predictive value in predicting RFS.

\section{The novel score contributes to treatment strategy selection of patients with BCLC 0 and $A$ stage}

Herein, we stratified HCC patients with BCLC 0 and A stage according to this novel score, and further compared the RFS of patients who underwent liver resection with that of patients who received LT (HCC-LT). 
Table 3 Operative data, postoperative complications and deaths of each groups in the training cohort

\begin{tabular}{|c|c|c|c|c|c|c|}
\hline \multirow[b]{2}{*}{ Variables } & \multicolumn{4}{|c|}{ Single segment-HCC $(n=151)$} & \multirow{2}{*}{$\begin{array}{l}\text { Multiple } \\
\text { segments-HCC } \\
(n=60)\end{array}$} & \multirow{2}{*}{$\begin{array}{l}\text { Single segment vs } \\
\text { Multiple segments } \\
\text { (P-value) }\end{array}$} \\
\hline & $\begin{array}{l}\text { Left segment } \\
(n=64)\end{array}$ & $\begin{array}{l}\text { Middle segment } \\
(\mathrm{n}=28)\end{array}$ & $\begin{array}{l}\text { Right segment } \\
(n=58)\end{array}$ & $\begin{array}{l}\text { Caudate area } \\
(n=1)\end{array}$ & & \\
\hline $\begin{array}{l}\text { Duration operation } \\
\text { (min) }\end{array}$ & $205.2 \pm 83.8$ & $201.4 \pm 70.3$ & $203.4 \pm 84.3$ & 620 & $241.0 \pm 86.5$ & 0.017 \\
\hline Blood loss (ml) & $283.5 \pm 356.8$ & $336.0 \pm 240.3$ & $321.6 \pm 312.1$ & 300 & $411.8 \pm 486.9$ & 0.185 \\
\hline Blood transfusion & & & & & & 0.635 \\
\hline Yes & $7(10.9 \%)$ & $9(32.1 \%)$ & $10(17.2 \%)$ & 0 & $12(20.0 \%)$ & - \\
\hline No & 57 (89.1\%) & 19 (67.9\%) & $48(82.8 \%)$ & $1(100.0 \%)$ & $48(80.0 \%)$ & - \\
\hline Surgical margin $(\mathrm{cm})$ & & & & & & 0.470 \\
\hline$<1.0$ & $20(31.3 \%)$ & $4(14.3 \%)$ & $9(15.5 \%)$ & 0 & $16(22.7 \%)$ & - \\
\hline$\geq 1.0$ & $44(68.7 \%)$ & $24(85.7 \%)$ & 49 (84.5\%) & $1(100.0 \%)$ & $44(77.3 \%)$ & - \\
\hline $\begin{array}{l}\text { Overall complica- } \\
\text { tions }\end{array}$ & $17(26.6 \%)$ & $7(25.0 \%)$ & $14(17.2 \%)$ & $0(0 \%)$ & $14(23.3 \%)$ & 0.781 \\
\hline $\begin{array}{l}\text { Wound infection } \\
\text { (Gradel) }\end{array}$ & $3(4.7 \%)$ & $1(3.6 \%)$ & $3(5.2 \%)$ & $0(0 \%)$ & $3(5.0 \%)$ & - \\
\hline Ascites (Gradel) & $9(14.1 \%)$ & $3(10.7 \%)$ & $8(13.8 \%)$ & $0(0 \%)$ & $6(10.0 \%)$ & - \\
\hline $\begin{array}{l}\text { Acute hepatic } \\
\text { failure (GradelV) }\end{array}$ & $1(1.6 \%)$ & $0(0 \%)$ & $0(0 \%)$ & $0(0 \%)$ & $1(1.7 \%)$ & - \\
\hline $\begin{array}{l}\text { Pulmonary inflam- } \\
\text { mation (Gradell) }\end{array}$ & $3(4.7 \%)$ & $3(10.7 \%)$ & $3(5.2 \%)$ & $0(0 \%)$ & $4(6.7 \%)$ & - \\
\hline $\begin{array}{l}\text { Haemorrhage } \\
\text { (Grade IV) }\end{array}$ & $1(1.6 \%)$ & $0(0 \%)$ & $0(0 \%)$ & $0(0 \%)$ & $0(0 \%)$ & - \\
\hline In-hospital mortality & $2(3.1 \%)$ & $0(0 \%)$ & $0(0 \%)$ & $0(0 \%)$ & $1(1.7 \%)$ & 0.850 \\
\hline
\end{tabular}

Clinicopathologic baseline data of HCC patients with BCLC 0 and A stage were illustrated in Table 4.

$64 \mathrm{HCC}$ patients with BCLC 0 and A stage were classified as score 0,13 as score 1,28 as score 2,17 as score 3, 3 as score 4,1 as score 5 . The median RFS of score 0 , score 1 , score 2 , score 3 , score 4 and score 5 group was 109.9 months (95\% CI, 99.8-120.0 months), 74.7 months (95\% CI, 51.0-98.5 months), 58.8 months (95\% CI, 38.479.3 months), 43.7 months (95\% CI, 24.4-63.0 months), 4.8 months (95\% CI, 1.9-7.8 months), and 23.8 months, respectively (Additional file 2: Figure S2A). Our data showed that there was no significant difference of RFS between patients with score 0 and liver transplantation recipients. The median RFS of patients with score 0 was better than that of patients with score $1-3$ and score 4-5 (108.1 months vs. 62.8 months, 108.1 months vs. 11.8 months, $P<0.05$ ) (Additional file 2: Figure S2B).

\section{Subgroup analysis: the novel score predicts RFS of patients with CLLTs}

Among 53 patients with CLLTs, there were 38 patients with HCC located in the single segment and 15 patients with HCC located in the multiple segments. There was no significant difference between the single segment group and the multiple segments group in clinical variables (Table 5).

Here, we evaluated the predictive value of the novel scoring system in predicting RFS of patients with CLLTs. According to the risk score, 23 patients with CLLTs were classified as score 0,10 as score 1,9 as score 2, 6 as score 3,3 as score 4,2 as score 5 . The median RFS of the patients with score 0 and score 1 , was 82.7 months (95\% CI, 64.0-101.4 months), and 62.1 months ( $95 \% \mathrm{CI}, 43.2-81.1$ months), respectively. More strikingly, the median RFS of score 2, score 3 , score 4 and score 5 group was 39.9 months (95\% CI, 14.1-65.7 months), 48.1 months (95\% CI, 26.869.3 months), 7.8 months (95\% CI, 4.3-11.2 months), and 16.1 months (95\% CI, 0.8-31.3 months), respectively (Fig. 1D). The median RFS of score $0-1$ and score $>1$ was 72.7 months (95\% CI, 61.9-83.5 months) and 53.0 months (95\% CI, 42.2-63.7 months), respectively (Fig. 1E). Additionally, we found that the AUCs of the novel score system at 1,3 , and 5 years were 0.637 , 0.646 , and 0.618 , respectively, and were greater than that of the other representative classification systems (Fig. 2G-I). 


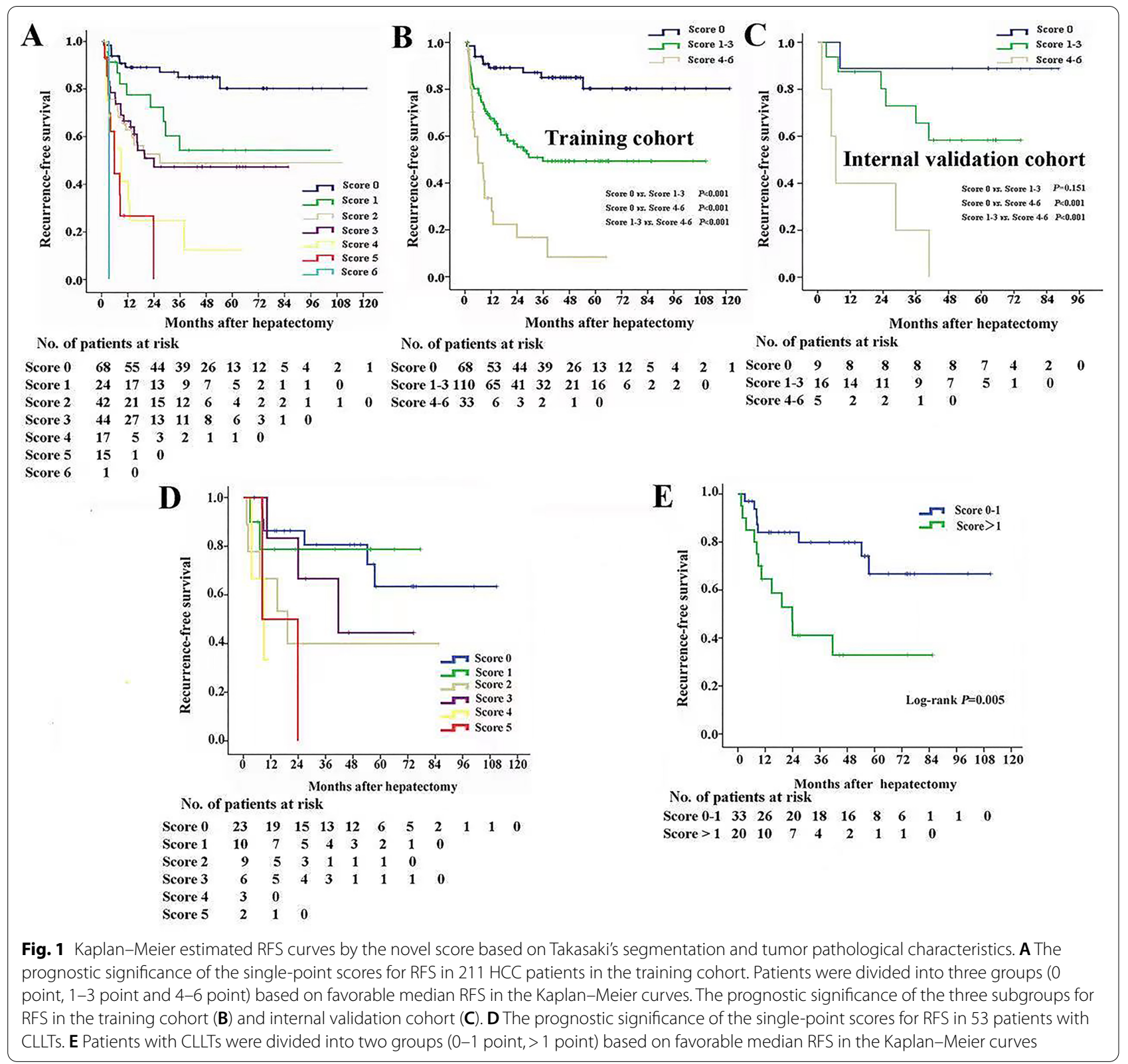

\section{Discussion}

Currently, many staging systems have been developed to classify patients with HCC. However, these classification systems for HCC (BCLC, HKLC and TNM) do not take account of tumor location. More strikingly, some previous studies found that the patients with multiple tumors located in the same lobe had higher RFS rates than patients with tumors located in different lobes after HR $[19,20]$. Consistently, Lv et al. indicated that in HCC patients with multifocal tumors meeting the Milan criteria, tumors located in the same hepatic section (Couinaud's segmentation) may lead to better long-term survival and lower $\mathrm{HCC}$ recurrence rates than those of tumors in different sections [9].

Obviously, these studies failed to clarify the impacts of the combination of anatomical sites and tumor biological characteristics on HCC recurrence after curative resection, and did not introduce a recurrence score system based on tumor location to guide anatomical liver resection. Herein, we constructed a novel score comprising tumor size, tumor location, MVI and differentiation grade, and the score allowed for a more accurate prognostic prediction for RFS of HCC patients with hepatectomies. In the present study, we first validated the 


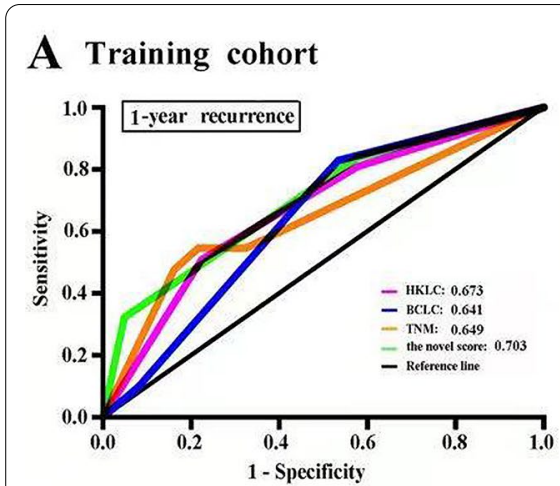

D Internal validation cohort
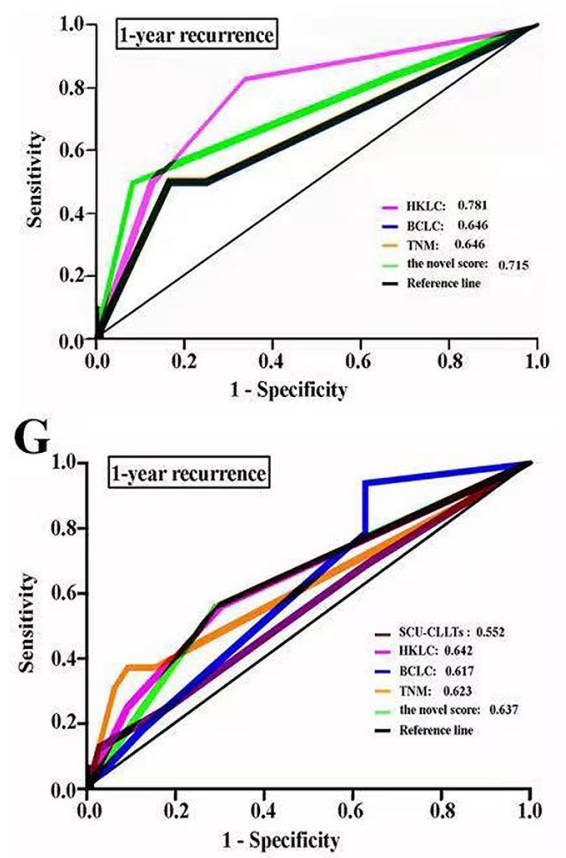

B

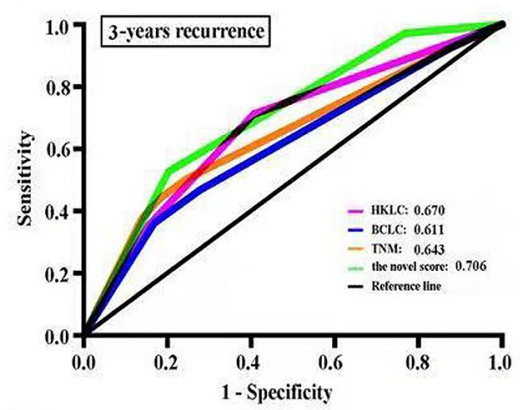

$\mathbf{E}$

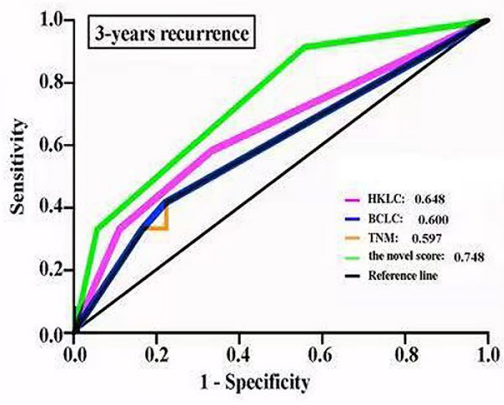

$\mathbf{H}_{1}$

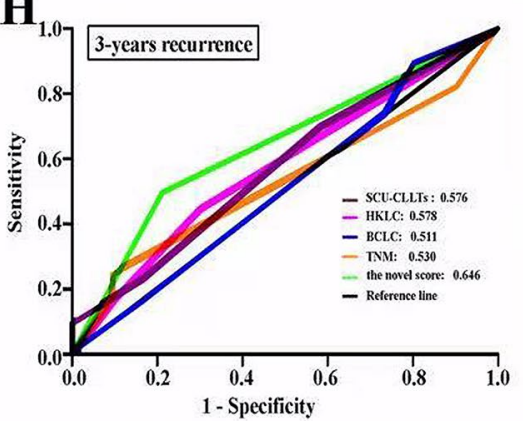

C

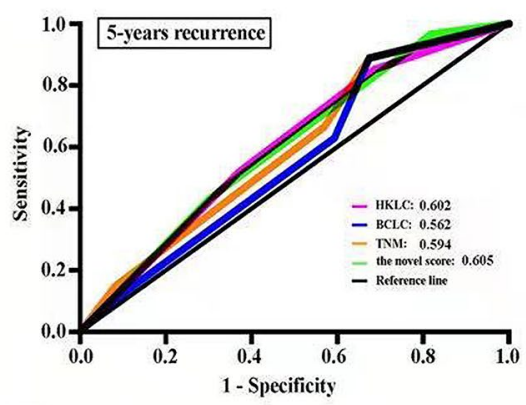

$\mathbf{F}$

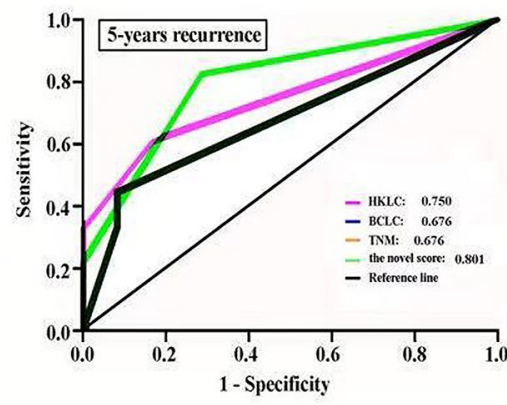

I

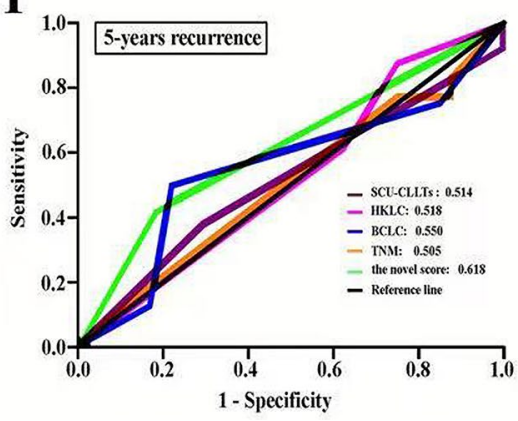

Fig. 2 The predictive accuracy of the novel score in HCC patients. The AUCS of the novel score and the representative classification systems (BCLC, TNM and HKLC) in predicting RFS of HCC patients at 1 year $(\mathbf{A}, \mathbf{D}), 3$ years $(\mathbf{B}, \mathbf{E})$ and 5 years $(\mathbf{C}, \mathbf{F})$ in the training cohort and validation cohort. The AUCs of the novel score and the representative classification systems (BCLC, TNM, HKLC and SCU-CLLTs) in predicting RFS of patients with CLLTs at 1 year $(\mathbf{G}), 3$ years $(\mathbf{H})$ and 5 years $(\mathbf{I})$

effect of tumor location (Takasaki's segmentation) on $\mathrm{HCC}$ recurrence, and demonstrated that $\mathrm{HCC}$ patients with located in single segment had significantly better RFS than patients with tumors located in multiple segments after HR. About $40 \%$ patients with tumors located in multiple segments had not less than 2 nodules, which may partly explain the high recurrence rates in patients with tumors located in multiple segments.

Nowadays, the BCLC system is widely used for prognosis prediction and treatment strategy selection [21, 22]. According to this criteria, RFA, HR and liver transplantation are recommended for early-stage tumors $(0$ and A stage). More significantly, this classification system performed well in stratifying patients with BCLC 0 and A stage relative to the RFS, which may provide prognostic data that are useful in the selection of surgical treatment. Our findings might support the notion that BCLC 0 and A stage HCC patients with low score are recommended as HR. As for BCLC 0 and A stage patients with high score, liver transplantation could be recommended.

Although a recent study from China proposed a classification system of CLLTs (SCU-CLLTs), which divided CLLTs into four subtypes based on anatomical location between lesions and hepatic principal vascular structures as well as the involvement of resected segments [18], the classification system did not take 
Table 4 Demographic characteristic of HCC patients with BCLC 0 and A stage

\begin{tabular}{llll}
\hline Variables & Hepatectomy $(\mathbf{n}=\mathbf{1 2 6})$ & LT $(\mathbf{n}=\mathbf{5 3})$ & $\boldsymbol{P}$-value \\
\hline Age (years) & $49.9 \pm 11.1$ & $49.9 \pm 11.2$ & 0.892 \\
Gender, M/F & $121(96.0 \%) / 5(4.0 \%)$ & $50(98.4 \%) / 3(1.6 \%)$ & 0.671 \\
AFP $(\mathrm{ng} / \mathrm{ml}), \leq 400 />400$ & $82(65.1 \%) / 34(34.9 \%)$ & $42(79.2 \%) / 11(20.8 \%)$ & 0.243 \\
HBV-DNA, \pm & $88(69.8 \%) / 38(30.1 \%)$ & $26(49.1 \%) / 27(50.9 \%)$ & 0.008 \\
Prothrombin time (s) & $13.6 \pm 1.2$ & $14.6 \pm 3.2$ & 0.001 \\
FIB-4 & $2.5 \pm 1.9$ & $2.7 \pm 1.5$ & 0.464 \\
Tumor size $(\mathrm{cm}),<5 / \geq 5$ & $76(60.3 \%) / 50(49.7 \%)$ & $28(52.8 \%) / 25(47.2 \%)$ & 0.354 \\
Tumor nodule number, Single / Multiple ( $\geq 2)$ & $122(96.8 \%) / 4(3.2 \%)$ & $50(94.4 \%) / 3(5.6 \%)$ & 0.433 \\
MVI, \pm & $14(11.1 \%) / 112(88.9 \%)$ & $16(30.2 \%) / 37(69.8 \%)$ & 0.002 \\
Differentiation grade ${ }^{a}$, I-II/III-IV & $118(93.7 \%) / 8(6.3 \%)$ & $47(88.7 \%) / 6(11.3 \%)$ & 0.258 \\
BCLC stage, O/A & $20(15.9 \%) / 106(84.1 \%)$ & $12(22.6 \%) / 41(77.4 \%)$ & 0.281 \\
\hline
\end{tabular}

HCC hepatocellular carcinoma, BCLC Barcelona Clinic Liver Cancer, LT liver transplantation, AFP alpha-fetoprotein, MVI microvascular invasion, $H C C$ hepatocellular carcinoma

${ }^{\text {a }}$ Edmondson-Steiner grade

Table 5 Demographic characteristic of 53 patients with CLLTs

\begin{tabular}{|c|c|c|c|}
\hline Variables & Single segment $(n=38)$ & Multiple segments $(n=15)$ & P-value \\
\hline Age (years) & $49.6 \pm 13.0$ & $55.0 \pm 14.2$ & 0.155 \\
\hline Gender, M:F & $37: 1$ & 15:0 & 0.526 \\
\hline Liver cirrhosis & & & 0.362 \\
\hline Present & $31(81.6 \%)$ & $12(80.0 \%)$ & - \\
\hline Absent & $7(18.4 \%)$ & $3(20.0 \%)$ & - \\
\hline $\operatorname{AFP}(\mathrm{ng} / \mathrm{ml})$ & $209.2 \pm 350.0$ & $246.0 \pm 409.3$ & 0.591 \\
\hline Tumor size $(\mathrm{cm})$ & & & 0.560 \\
\hline$<5$ & $26(68.4 \%)$ & $9(66.7 \%)$ & - \\
\hline$\geq 5$ & $12(31.6 \%)$ & $6(33.3 \%)$ & - \\
\hline Tumor nodule number & & & 0.986 \\
\hline Single & $33(86.8 \%)$ & $13(86.7 \%)$ & - \\
\hline Multiple ( $\geq 2$ ) & $5(13.2 \%)$ & $2(13.3 \%)$ & - \\
\hline MVI & & & 0.531 \\
\hline Present & $5(13.2 \%)$ & $3(20.0 \%)$ & - \\
\hline Absent & $33(86.8 \%)$ & $12(80.0 \%)$ & - \\
\hline Differentiation grade & & & 0.879 \\
\hline$|-| \mid$ & $35(92.1 \%)$ & $14(93.3 \%)$ & - \\
\hline III-IV & $3(7.9 \%)$ & $1(6.7 \%)$ & - \\
\hline Surgical methods & & & 0.328 \\
\hline Mesohepatectomy & $10(26.3 \%)$ & $6(100 \%)$ & - \\
\hline Extended left/right hepatectomy & $28(73.7 \%)$ & $9(0 \%)$ & - \\
\hline Duration operation (min) & $197.1 \pm 75.7$ & $223.4 \pm 49.6$ & 0.291 \\
\hline Blood loss (ml) & $296.0 \pm 220.9$ & $403.6 \pm 621.6$ & 0.362 \\
\hline Surgical margin $(\mathrm{cm})$ & & & 0.713 \\
\hline$<1.0$ & $6(15.8 \%)$ & $3(20.0 \%)$ & - \\
\hline$\geq 1.0$ & $32(84.2 \%)$ & $12(80.0 \%)$ & - \\
\hline Overall complications & $9(23.7 \%)$ & $4(26.7 \%)$ & 0.820 \\
\hline Ascites (Grade I) & $5(13.2 \%)$ & $3(20.0 \%)$ & - \\
\hline Pulmonary inflammation (Grade II) & $3(7.9 \%)$ & $1(6.7 \%)$ & - \\
\hline Wound infection (Grade I) & $1(2.6 \%)$ & $0(0 \%)$ & - \\
\hline In-hospital mortality & $0(0 \%)$ & $0(0 \%)$ & - \\
\hline
\end{tabular}


account of parameters such as the distance from the tumors to important structures and tumor size. In our study, our data demonstrated that the novel score system was a reliable classification system of $\mathrm{HCC}$ patients with CLLTs, and had a better predictive value for RFS compared to the representative classification systems. An increased predictive accuracy of our novel score is due to the fact that the recurrence of HCC with CLLTs depends on the contribution and interaction of tumor biology characteristics and tumor location (Takasaki's segmentation).

Clinically, anatomical resection using Takasaki's Glissonean pedicle transection method is widely performed for two decades in hepatocellular carcinoma (HCC) [14]. The Glissonean pedicle approach has provided in-depth knowledge of the surgical anatomy of the liver and has made different types of hepatectomy. Notably, in this study, we validated the impact of tumor location (Takasaki's segmentation) on HCC recurrence, and sought to introduce a recurrence score system based on tumor location (Takasaki's segmentation) to guide anatomical liver resection using Takasaki's Glissonean pedicle transection method. Thus, we proposed a novel classification system of CLLTs based on tumor location (Takasaki's segmentation) and tumor biology characteristics. As was shown in Additional file 5: Table S3, our novel classification system of CLLTs may help to define the extent of resection, provide a prognostic assessment, and guide the precision hepatectomy for CLLTs. Three subtypes of CLLTs were presented as following: tumors arising from the liver parenchyma of Couinaud's segment $\mathrm{V}$ and/or $\mathrm{VIII} \pm \mathrm{I}$ with score $0-1$ were classified as type A lesions. These lesions required anatomical resection of the middle segment \pm the caudate. Tumors arising from the liver parenchyma of Couinaud's segment IV with score 0-1 were classified as type B lesions. These lesions required anatomical resection of Couinaud's segment IV. Lesions arising from multiple segments, and tumors located within CLLTs with score $>1$ were classified as type C. For patients with type $\mathrm{C}$, Glissonean pedicle transection method for mesohepatectomy $(\mathrm{MH})$ is performed conventionally to achieve curative resection. However, $\mathrm{MH}$ is not recommended as the surgical therapy for patients with type I and type II in the SCU-CLLTs classification system, which may partly explain why there is no significant difference in RFS among the four subtypes [23].

In line with the previous findings [18, 24], our data demonstrated that there were no differences in the perioperative death and postoperative complications of patients with CLLTs who underwent $\mathrm{MH}$ or extended left/right hepatectomy. These findings led us to conclude that the improvements in surgical techniques, low central venous pressure maintenance and the application of surgical energy platform allow $\mathrm{MH}$ to be a safe and feasible choice for patients with CLLTs.

The main limitation of our study is that our classification system of HCC patients came from a single institution in China where hepatitis B is prevalent. The classification system is needed to further verify in other centers. Furthermore, the present study is a small-scale retrospective study, limited by the inherent defects of the analysis.

\section{Conclusion}

In this study, we developed and validated a novel score classification for predicting the RFS of the Asian patients who received HR for HCC. The combination of anatomical sites (Takasaki's segmentation) and tumor biological characteristics could provide an accurate individualized estimation of recurrence, and may help to select patients with a less favorable prognosis for adjuvant or alternative therapies. Furthermore, our findings highlighted the value of tumor location (Takasaki's segmentation) in the assessment of precision hepatectomy for patients with BCLC 0 and A stage, and CLLTs.

\section{Abbreviations \\ HCC: Hepatocelluar carcinoma; RFS: Recurrence-free survival; AUCs: Area under the receiver operating characteristic curves; CLLTs: Cen- trally located liver tumors; HBV: Hepatitis B virus; HCV: Hepatitis C virus; HR: Hepatic resection; LT: Liver transplantation; CT: Computed tomography; MRI: Magnetic resonance imaging; AFP: $a$-Fetoprotein; RFA: Radiofrequency abla- tion; TACE: Transcatheter arterial chemoembolization; HRs: Hazard ratios; Cls: Confidence intervals; BCLC: Barcelona Clinic Liver Cancer; HKLC: HongKong Liver Cancer; AJCC: American Joint Committee on Cancer; MH: Mesohepatec- tomy; MVI: Microvascular invasion.}

\section{Supplementary Information}

The online version contains supplementary material available at https://doi. org/10.1186/s12893-021-01275-3.

Additional file 1: Figure S1. The recurrence rates of HCC patients who underwent liver resection in four subgroups. Showing a significant lower recurrence rates in the middle segment group compared with the other groups.

Additional file 2: Figure S2. Curves of RFS for HCC patients with BCLC 0 and A stage after hepatectomy and LT. The prognostic significance of the single-point scores for RFS in $126 \mathrm{HCC}$ patients with BCLC 0 and A stage who underwent hepatectomy $(\mathbf{A})$. Patients with BCLC 0 and A stage underwent hepatectomy were divided into three groups (0 point, 1-3 point, and 4-5 point) based on favorable median RFS in the Kaplan-Meier curves (B)

Additional file 3: Table S1. Recurrence-free survival data for HCC patients.

Additional file 4: Table S2. Univariate and multivariate Cox analysis of risk factors predicting recurrence-free survival of HCC in the training cohort.

Additional file 5: Table S3. Classification of CLLTS. 


\section{Authors' contributions}

All authors critically revised the manuscript, approved the final version to be published, and agree to be accountable for all aspects of the work. Concept and design of the study: WQ, YY. Administrative support: WQ, YY. Data collection and acquisition: WQ, LW, HT, CCX and HXL. Data analysis: LW, BYH, and HT. Manuscript preparation: WQ, LW, BYH, HT and YY.

\section{Funding}

This study was supported by the grants from the National Key Research and Development Program (2017YFA0104304) and China Postdoctoral Science Foundation (2017M611459). The funding body had no role in the design of the study and collection, analysis, and interpretation of the data, preparation, review, or approval of the manuscript, and decision to submit the manuscript for publication.

\section{Availability of data and materials}

The data that support the findings of this study are available on request from the corresponding author. The data are not publicly available due to privacy or ethical restrictions.

\section{Declarations}

\section{Ethics approval and consent to participate}

This study was approved by the Ethics Committee of the Third Affiliated Hospital of Sun Yat-sen University, and conducted in accordance with the Declaration of Helsinki. All patients signed informed consent that data and follow-up will be collected and is potentially used for scientific analysis.

\section{Consent for publication}

Not applicable.

\section{Competing interests}

All authors declare no conflict of interest for this article.

\section{Author details}

${ }^{1}$ Department of Hepatic Surgery, The Third Affiliated Hospital of Sun Yat-Sen University, 600 Tianhe Road, Guangzhou 510630, China. ${ }^{2}$ Department of General Surgery, Huashan Hospital, Cancer Metastasis Institute, Fudan University, 12 Urumqi Road (M), Shanghai 200040, China. ${ }^{3}$ Department of Breast Surgery, Breast Tumor Center, Sun Yat-Sen Memorial Hospital, Sun Yat-Sen University, 107 Yanjiang West Road, Guangzhou 510235, China. ${ }^{4}$ Guangdong Provincial Key Laboratory of Liver Disease Research, 600 Tianhe Road, Guangzhou 510630, China.

Received: 11 November 2020 Accepted: 26 May 2021

Published online: 03 June 2021

\section{References}

1. Siegel R, Ma J, Zou Z, Jemal A. Cancer statistics, 2014. CA Cancer J Clin. 2014;64(1):9-29.

2. Perz JF, Armstrong GL, Farrington LA, Hutin YJ, Bell BP. The contributions of hepatitis $B$ virus and hepatitis $C$ virus infections to cirrhosis and primary liver cancer worldwide. J Hepatol. 2006;45(4):529-38.

3. Fan ST, Lo CM, Liu CL, Lam CM, Yuen WK, Yeung C, et al. Hepatectomy for hepatocellular carcinoma: Toward zero hospital deaths. Ann Surg. 1999;229(3):322-30.

4. Colella G, Bottelli R, De Carlis L, Sansalone CV, Rondinara GF, Alberti A, et al. Hepatocellular carcinoma: Comparison between liver transplantation, resective surgery, ethanol injection, and chemoembolization. Transpl Int. 1998;11(Suppl 1):S193-6.

5. Fong Y, Sun RL, Jarnagin W, Blumgart LH. An analysis of 412 cases of hepatocellular carcinoma at a Western center. Ann Surg. 1999;229(6):790-9.
6. Zhang H, Han J, Xing H, Li ZL, Schwartz ME, Zhou YH, et al. Sex difference in recurrence and survival after liver resection for hepatocellular carcinoma: a multicenter study. Surgery. 2019;165(3):516-24.

7. Regimbeau JM, Kianmanesh R, Farges O, Dondero F, Sauvanet A, Belghiti J. Extent of liver resection influences the outcome in patients with cirrhosis and small hepatocellular carcinoma. Surgery. 2002;131(3):311-7.

8. Qin W, Wang L, Hu B, Leng S, Tian H, Luo H, et al. A novel score predicts HBV-related hepatocellular carcinoma recurrence after hepatectomy: a retrospective multicenter study. J Gastrointest Surg. 2019;23(5):922-32.

9. Lv T, Jiang L, Yan L, Yang J, Li B, Wen T, et al. Multiple tumors located in the same section are associated with better outcomes after hepatic resection for HCC Patients Meeting the Milan Criteria. J Gastrointest Surg. 2015;19(12):2207-14

10. Takasaki K. Glissonean pedicle transection method for hepatic resection: a new concept of liver segmentation. J Hepatobiliary Pancreat Surg. 1998;5(3):286-91.

11. Yamamoto M, Katagiri S, Ariizumi S, Kotera Y, Takahashi Y. Glissonean pedicle transection method for liver surgery (with video). J Hepatobiliary Pancreat Sci. 2012;19(1):3-8.

12. Kolly P, Reeves H, Sangro B, Knöpfli M, Candinas D, Dufour JF. Assessment of the Hong Kong Liver Cancer staging system in Europe. Liver Int. 2016;36(3):911-7.

13. Yan X, Fu X, Cai C, Zi X, Yao H, Qiu Y. Validation of models in patients with hepatocellular carcinoma: comparison of Hong Kong Liver Cancer with Barcelona Clinic Liver Cancer staging system in a Chinese cohort. Eur J Gastroenterol Hepatol. 2015;27(10):1180-6.

14. Yang T, Zhang J, Lu JH, Yang LQ, Yang GS, Wu MC, et al. A new staging system for resectable hepatocellular carcinoma: comparison with six existing staging systems in a large Chinese cohort. J Cancer Res Clin Oncol. 2011;137(5):739-50.

15. Bruix J, Sherman M. American Association for the Study of Liver Diseases, Management of hepatocellular carcinoma: an update. Hepatology. 2011;53(3):1020-2.

16. Kishi Y, Abdalla EK, Chun YS, Zorzi D, Madoff DC, Wallace MJ, et al. Three hundred and one consecutive extended right hepatectomies: evaluation of outcome based on systematic liver volumetry. Ann Surg. 2009;250(4):540-8.

17. Chen XP, Qiu FZ, Lau WY, Zhang BX, Chen YF, Zhang WG, et al. Mesohepatectomy for hepatocellular carcinoma: a study of 256 patients. Int J Colorectal Dis. 2008;23(5):543-6.

18. Qiu J, Wu H, Bai Y, Xu Y, Zhou J, Yuan H, et al. Mesohepatectomy for centrally located liver tumours. Br J Surg. 2013;100(12):1620-6.

19. Feng K, Yan J, Li X, Xia F, Ma K, Wang S, et al. A randomized controlled trial of radiofrequency ablation and surgical resection in the treatment of small hepatocellular carcinoma. J Hepatol. 2012;57(4):794-802.

20. Jiang L, Liao A, Wen T, Yan L, Li B, Yang J, et al. Living donor liver transplantation or resection for Child-Pugh A hepatocellular carcinoma patients with multiple nodules meeting the Milan criteria. Transpl Int. 2014;27(6):562-9.

21. Forner A, Llovet JM, Bruix J. Hepatocellular carcinoma. Lancet. 2012:379(9822):1245-55.

22. Llovet JM, Di Bisceglie AM, Bruix J, Kramer BS, Lencioni R, Zhu AX, et al. Design and endpoints of clinical trials in hepatocellular carcinoma. J Natl Cancer Inst. 2008;100(10):698-711.

23. Qiu J, Chen S, Wu H, Dou CY. The prognostic value of a classification system for centrally located liver tumors in the setting of hepatocellular carcinoma after mesohepatectomy. Surg Oncol. 2016;25(4):441-7.

24. Yang LY, Chang RM, Lau WY, Ou DP, Wu W, Zeng ZJ. Mesohepatectomy for centrally located large hepatocellular carcinoma: indications, techniques, and outcomes. Surgery. 2014;156(5):1177-87.

\section{Publisher's Note}

Springer Nature remains neutral with regard to jurisdictional claims in published maps and institutional affiliations. 